https://doi.org/10.30843/nzpp.2018.71.209

\title{
Eucalyptus variegated beetle: genetic diversity of New Zealand and Australian collections
}

\author{
Aymee S.R. Lewis ${ }^{1, \star}$, Toni M. Withers ${ }^{1}$, Helen F. Nahrung ${ }^{2}$, Rebecca L. McDougal ${ }^{1}$, Chris A.M. Reid ${ }^{3}$ \\ and Geoff R. Allen ${ }^{4}$ \\ ${ }^{1}$ Scion, Private Bag 3020, Rotorua 3046, New Zealand \\ ${ }^{2}$ University of the Sunshine Coast, Maroochydore DC, QLD 4558, Australia \\ ${ }^{3}$ Australian Museum, 1 William Street, Sydney, NSW 2010, Australia \\ ${ }^{4}$ University of Tasmania, Private Bag 98, Hobart, TAS 7001, Australia \\ ${ }^{*}$ Corresponding author: Aymee.Lewis@scionresearch.com
}

Paropsisterna variicollis, the eucalyptus variegated beetle, was first detected in New Zealand in 2016. It threatens a growing eucalyptus forestry sector through larvae and adults causing significant defoliation to important plantation species. This work aimed to clarify the identification and origin of the New Zealand incursion to inform selection of suitable biological control agent(s). Australian and New Zealand specimens from the Paropsisterna obovata-variicollis-cloelia species complex were analysed by PCR and sequencing of two genetic loci, cytochrome c subunit I (COI) and cytochrome b (Cyt b). Molecular analysis of both genetic regions showed three major clusters of diversity. Cluster 1, proposed as Paropsisterna variicollis, had maximum 1.3\% genetic variation and was collected from New Zealand, Western Australia and from geographically diverse locations in eastern Australia. Taxonomic results identified distinctive phenotypes of other closely related beetle species, assisting in proposing Cluster 2 as Paropsisterna near decolorata and Cluster 3 as Paropsisterna agricola. Molecular results were compared to morphological structures on adult beetles.

https://doi.org/10.30843/nzpp.2018.71.199

\section{Parasitoids associated with codling moth (Cydia pomonella) in apple- growing regions in New Zealand}

\author{
Vicky A. Davis*, W.R. Manoharie Sandanayaka and John G. Charles \\ The New Zealand Institute for Plant \& Food Research Ltd, Private Bag 92169, Auckland 1142, New Zealand \\ ${ }^{*}$ Corresponding author: Vicky.Davis@plantandfood.co.nz
}

Codling moth (Cydia pomonella) (CM) is a major pest of apples in New Zealand. Several biocontrol agents introduced in the past to control CM have only been partially successful at reducing CM populations, so a parasitoid wasp, Mastrus ridens (Hymenoptera: Ichneumonidae), was recently released into apple-growing regions. This study sought evidence of the establishment of CM parasitoids. Corrugated cardboard bands ( 2 - $\mathrm{cm}$ wide bands with sentinel CM larvae and $10-\mathrm{cm}$ wide empty bands to trap wild CM larvae) were used to assess the presence of $M$. ridens and other CM parasitoids in Hawke's Bay, Nelson, Central Otago and Waikato regions. Five CM parasitoid species, including M. ridens, were recovered from sentinel and wide bands. Liotryphon caudatus (Hymenoptera: Ichneumonidae, released to control CM in 1906) was found in Hawke's Bay and Waikato. Ascogaster quadridentata (Hymenoptera: Braconidae, released to control CM in the 1930s), Glabridorsum stokesii (Hymenoptera: Ichneumonidae, released in 1967 to control light brown apple moth), Dibrachys microgastri (Hymenoptera: Chalcidoidea: Pteromalidae, an accidental arrival) and $M$. ridens were found in all regions. The interspecific competition between $M$. ridens and other parasitoid species remains to be investigated. 